\title{
Spatial convolution and correlation of optical fields via degenerate four-wave mixing
}

\author{
David M. Pepper, John AuYeung, Dan Fekete, and Amnon Yariv \\ California Institute of Technology, Pasadena, California 91125 \\ Received March 13, 1978
}

\begin{abstract}
A nonlinear optical technique is described that performs, essentially instantaneously, the functions of spatial correlation and convolution of spatially encoded waves. These real-time operations are accomplished by mixing spatially dependent optical fields in the Fourier-transform plane of a lens system. The use of a degenerate four-wave mixing scheme eliminates (in the Fresnel approximation) phase-matching restrictions and (optical) frequency-scaling factors. Spatial bandwidth-gain considerations and numerical examples, as well as applications to nonlinear microscopy, are presented.
\end{abstract}

In recent years, coherent and incoherent image processing has been demonstrated in a variety of applications, including pattern recognition, guidance systems, and other data-processing techniques. Present methods used to generate convolution and correlation operations of spatially encoded optical images include digital processing and Van der Lugt-type holograms..$^{1,2}$

It has been proposed by one of the authors (A. Yariv) that nonlinear optical techniques can be used to perform real-time holographic functions. ${ }^{3,4}$ The specific application of nonlinear three-wave mixing to perform the operation of convolution and correlation has been proposed by Eremeeva et al. ${ }^{5}$ The proposed scheme was demonstrated in the case of simple (luminous spot) images. These schemes, as well as that proposed in Refs. 3 and 4, suffered from restrictions on the spatial bandwidth of the information, which were due to the need for phase matching. Second, the use of multiple wavelengths introduces spatial scaling that may be objectionable.

In the analysis that follows we propose the use of four-wave mixing for real-time correlation and convolution operations. The process of four-wave mixing, which has recently been applied to the problem of "time-reversed" propagation, 6,7 is shown to be free of the phase-matching problem and to require a single frequency.

Consider the nature of the field produced as a result of the simultaneous mixing of three optical fields, all of radian frequency $\omega$, incident upon a thin medium possessing a third-order nonlinear optical susceptibility, $\chi_{N L}{ }^{(3)}$, centered at the common focal plane of two identical lenses (or mirrors) of focal length $f$. The geometry is illustrated in Fig. 1. Each field is specified spatially at the front focal plane of its respective lens with the following amplitudes:

$$
\begin{gathered}
E_{1}=\frac{1}{2} A_{1}(x, y, z) \exp [i(k z-\omega t)]+\text { c.c. }, \\
E_{2}=\frac{1}{2} A_{2}(x, y, z) \exp [-i(k z+\omega t)]+\text { c.c., }
\end{gathered}
$$

$$
E_{4}=\frac{1}{2} A_{4}(x, y, z) \exp [i(k z-\omega t)]+\text { c.c., }
$$

where $A_{1,4}(x, y, 0) \equiv u_{1,4}(x, y)$ and $A_{2}(x, y, 4 f) \equiv u_{2}(x, y)$. Fields $E_{1}$ and $E_{2}$ are essentially counterpropagating, with $E_{4}$ being parallel to these fields and separated either spatially [e.g., shifted by $\left.\left(x_{s}, y_{s}\right)\right]$ or via orthogonal polarizations. The $u_{i}$ contain the input information to be convolved or correlated, which can, for example, be in the form of phase and/or amplitude transparencies. The $u_{i}$ are assumed to be illuminated by plane waves, all of the same frequency, $\omega$.

After propagating through lens $L_{1}, A_{1}$ has the following form (in the Fresnel approximation),

$$
\begin{aligned}
& A_{1}(x, y ; f \leq z \leq 3 f)=\frac{\exp (i k n \Delta) \exp (i k z)}{i \lambda f} \\
& \quad \times \mathscr{F}\left\{u_{1}\left(x^{\prime}, y^{\prime}\right) \exp \left[\frac{i k}{2 f}\left(2-\frac{z}{f}\right)\left(x^{\prime 2}+y^{\prime 2}\right)\right]\right\},
\end{aligned}
$$

where $\mathfrak{F}\{a\} \equiv \tilde{a}$ is the Fourier transform of $a$, and

$$
\exp (i k n \Delta) \exp \left[-\frac{i k}{2 f}\left(x^{2}+y^{2}\right)\right]
$$

is the transmission function of a thin lens. ${ }^{2}$ The argu-

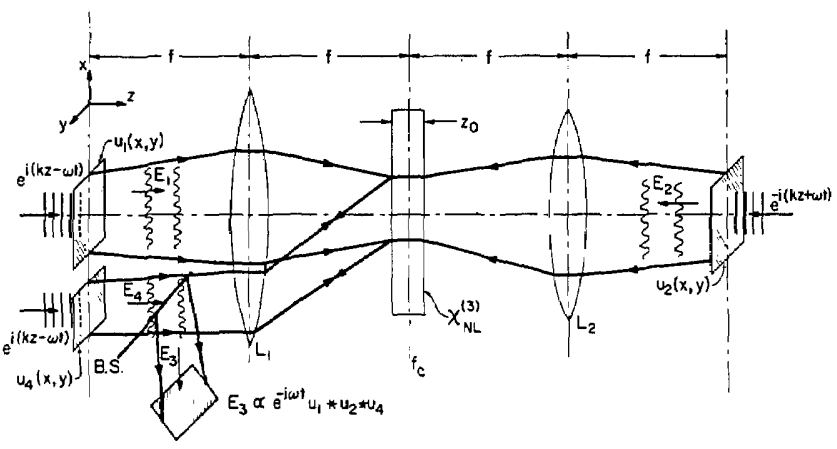

Fig. 1. Convolution/correlation geometry. All input optical fields are at frequency $\omega$. BS is a beam splitter necessary to view the desired output, $E_{3}$, which is evaluated at a plane located a distance $f$ from the lens $L_{1}$. 
ments of the Fourier transform are $f_{x}=x / \lambda f$ and $f_{y}=$ $y / \lambda f$, where $f_{x}$ and $f_{y}$ are the spatial frequencies in the $x$ and $y$ directions, respectively; in Eq. (3), $\Delta$ corresponds to the thickness of the lens. Similarly, the complex amplitude of $E_{2}$ has the form (after propagating through lens $\mathrm{L}_{2}$ ),

$$
\begin{aligned}
& A_{2}(x, y ; f \leq z \leq 3 f)=\frac{\exp (i k n \Delta) \exp [i k(4 f-z)]}{i \lambda f} \\
& \times \mathfrak{F}\left\{u_{2}\left(x^{\prime}, y^{\prime}\right) \exp \left[-\frac{i k}{2 f}\left(2-\frac{z}{f}\right)\left(x^{\prime 2}+y^{\prime 2}\right)\right]\right\} .
\end{aligned}
$$

Anticipating the mixing process of interest, we express the complex conjugate amplitude of $E_{4}$ (after propagating through lens $L_{1}$ ) as

$$
\begin{aligned}
& A_{4} *(x, y ; f \leq z \leq 3 f)=\frac{\exp (-i k n \Delta) \exp (-i k z)}{-i \lambda f} \\
& \times\left(\mathcal{F}\left\{u_{4}\left(x^{\prime}, y^{\prime}\right) \exp \left[\frac{i k}{2 f}\left(2-\frac{z}{f}\right)\left(x^{\prime 2}+y^{\prime 2}\right)\right]\right\}\right)^{*} .
\end{aligned}
$$

The fields $A_{\mathrm{i}}$ in Eqs. (2), (4), and (5) become directly proportional to the Fourier transforms of their respective $u_{i}$ if the terms inside the square brackets of the exponents can be neglected. This happens when

$$
|z-2 f| \ll 2 f^{2} \lambda / r_{i}^{2},
$$

where $r_{i}$ is the spatial extent of $u_{i}$.

We now place a medium centered at the focal plane $f_{c}$ of thickness $z_{0}$ [satisfying the constraint (6)], which possesses a third-order nonlinear optical susceptibility, $\chi_{\mathrm{NL}}{ }^{(3)}$. Without loss of generality, we assume a transparent, lossless medium and neglect linear refractive effects.

The complex amplitude of nonlinear polarization at $\omega=\omega+\omega-\omega$ generated by the mixing of the three waves is 8

$$
P_{\mathrm{NL}_{i}}=\chi_{i j k l}{ }^{(3)} A_{1 j} A_{2 k} A_{4_{l}}{ }^{*},
$$

where repeated indices are summed over the field polarizations. The resultant field, $E_{3}$, which satisfies both photon energy and momentum conservation, is given by

$$
A_{3}(\mathbf{x})=-\frac{4 \pi \omega^{2}}{c^{2}} \int P_{\mathrm{NL}}\left(\mathbf{x}^{\prime}\right) G\left(\mathbf{x}, \mathbf{x}^{\prime}\right) \mathrm{d}^{3} \mathbf{x}^{\prime},
$$

where

$$
G\left(\mathbf{x}, \mathbf{x}^{\prime}\right)=-\frac{1}{4 \pi} \frac{\exp (i k r)}{r}
$$

is the Green's function that satisfies the wave equation. Using Eqs. (2), (4), and (5) in Eqs. (7) and (8), as well as condition (6), integrating over the volume of the nonlinear medium, and "propagating" back to $z=0$ through lens $L_{1}$, we get

$$
\begin{gathered}
A_{3}\left(x_{0}, y_{0}, 0\right)=-i \frac{2 \pi \omega}{c} \frac{z_{0}}{\lambda^{4} f^{4}} \exp (2 i k n \Delta) \exp (4 i k f) \\
\times \mathcal{F}\left\{\tilde{u}_{1}(x, y) \tilde{u}_{2}(x, y) \tilde{u}_{4}^{*}(x, y) \chi_{\mathrm{NL}}{ }^{(3)}(x, y)\right\}
\end{gathered}
$$

where $\chi_{\mathrm{NL}}{ }^{(3)}(x, y)$ is the proper tensor element in Eq. (7) connecting fields $1,2,3$, and 4 .

If $\chi_{\mathrm{NL}}{ }^{(3)}$ is spatially homogeneous, the output field can be written in the form

$$
\begin{aligned}
& A_{3}\left(x_{0}, y_{0}, 0\right) \\
& \quad=\psi u_{1}(-x,-y) * u_{2}(-x,-y) \star u_{4}(-x,-y),
\end{aligned}
$$

where

$$
\psi=-i \frac{2 \pi \omega}{c} \frac{z_{0}}{\lambda^{4} f^{4}} \exp (2 i k n \Delta) \exp (4 i k f) \chi_{\mathrm{NL}}{ }^{(3)} .
$$

In Eq. (11) the symbols $*$ and $\star$ denote the standard operations of convolution and correlation, respectively. ${ }^{2}$

Equation (11) is our primary result. We obtain the spatial convolution of $u_{1}$ and $u_{2}$ by taking $u_{4}$ as a point source. This leads to

$$
A_{3}\left(x_{0}, y_{0}, 0\right)=\psi u_{1} * u_{2} .
$$

Similarly, the correlation operation is performed by placing information on fields $u_{1}\left(u_{2}\right)$ and $u_{4}$, with a point source for $u_{2}\left(u_{2}\right)$ yielding

$$
A_{3}\left(x_{0}, y_{0}, 0\right)=\psi u_{1} \star u_{4},
$$

where the $u_{i}$ in Eqs. (13) and (14) are the inverted images of the input fields [compare Eq. (11)].

We can now appreciate the advantage of using a degenerate four-wave mixing approach to real-time operations. The third field (which corresponds to the point-source input mentioned above) provides an optical carrier frequency upon which the convolution or correlation information is placed. No frequency-scaling factors are present (compare Ref. 5), the entire system requires only a single frequency source, and within the Fresnel approximation the phase-matching condition is satisfied. Finally, the "degenerate" operations of autoconvolution and autocorrelation can be performed with a single optical frequency.

The approximations used in the above discussion place upper limits on both the resolution (or spatial frequency bandwidth) and the efficiency (or nonlinear gain) of the interaction. The Fresnel approximation is related to $f_{\max }$, the greatest spatial frequency present, by

$$
f_{\max }<\left(4 / \pi \lambda^{3} f\right)^{1 / 4} .
$$

This same approximation also places an upper limit on the input field spot size, which is given by

$$
d<\left(4 \lambda f^{3 / \pi}\right)^{1 / 4} .
$$

Hence, the maximum number of resolution elements possible is derivable through Eqs. (15) and (16), yielding

$$
N_{\max }=16 f / \pi \lambda
$$

Using values of $10 \mathrm{~cm}$ and $0.5 \mu \mathrm{m}$ for $f$ and $\lambda$, respectively, leads to a value of $10^{3} \mathrm{~cm}^{-1}$ for $f_{\max }$, which corresponds to a grid of $1000 \times 1000$ resolution elements. 
Therefore, since the phase-matching condition is satisfied for all the field momentum components in the Fresnel approximation, this technique can be useful for complex spatial-information processing.

The second constraint, (6), yields an upper limit to the nonlinear gain of the interaction. From (6), we obtain

$$
z_{0} \ll b_{0}=2 \pi^{2} \omega_{0}^{2} / \lambda,
$$

where $\omega_{0}$ is the spot size in the focal plane. For Gaussian beams, the value of $b_{0}$ corresponds to $2 \pi$ times the confocal parameter. ${ }^{9}$ The spot size in the focal plane of the Gaussian "point source" (or the field corresponding to the optical carrier frequency) determines the maximum spatial frequency of the convolution or correlation operation. It can be shown that the number of resolution elements is

$$
N \approx\left(d_{1} / d_{2}\right)^{2},
$$

where $d_{1}$ is the spatial extent of the input field and $d_{2}$ is the input-aperture size of Gaussian "point source," assuming that both are satisfying the Fresnel approximation, Eq. (16). It follows that, for a given input power and choice of lens focal length, the output power with information present in the two input fields is related to that for no information present by

$$
P_{\text {out }_{\text {info }}} \approx P_{\text {out }_{\text {no info }}} / N .
$$

Thus we see that there is a tradeoff between the spatial bandwidth product, $N$, and the output power (or nonlinear gain) of the interaction. For the lens and optical wavelength used above, we find for $\mathrm{CS}_{2}$ (using a value of $\chi^{(3)} \sim 1.8 \times 10^{-12}$ e.s.u.) that $P_{\text {out info }} \sim 200 \mathrm{~W}$ for $P_{1,2,4} \sim 0.5 \mathrm{MW}$, with $d_{1}=1 \mathrm{~cm}$ and $d_{2}=0.01 \mathrm{~cm}$. This corresponds to $N \sim 10^{4}$.

We note that the output efficiency increases nonlinearly ${ }^{7}$ as

$$
P_{\text {out }} \sim P_{\text {in }} \tan ^{2}(|\kappa| L),
$$

which has been recently verified experimentally. ${ }^{10}$ However, since the desired convolution or correlation operations necessarily require an output field given by Eq. (10), the small-angle approximation to $\tan (|\kappa| L)$ must be valid, which has also been verified experimentally. ${ }^{10,11}$ Hence, as the nonlinear gain, $|\kappa| L$, becomes larger, and the higher-order terms contained in the power series expansion of Eq. (21) become appreciable, distortions in the desired output operations result. We are thus limited to a nonlinear interaction where the amplitude changes for all the input fields are minimal (which is satisfied in the above numerical example).

This interaction operationally corresponds to a real-time holographic analog 12 of a Van der Lugt filter system. The extension of these concepts to real-time matched filters, pattern recognition, and other forms of image processing follows directly. The implementation of this scheme to integrated optics using geodesic lenses ${ }^{13}$ or graded fibers ${ }^{4}$ is a viable possibility, considering the increased intensities present in optical waveguides. We have recently ${ }^{14}$ proposed and analyzed the possibility of performing real-time holography by four-wave mixing in optical waveguides. The extension of that analysis to the present set of operations easily follows. Further, this method can be applied to the field of nonlinear microscopy. ${ }^{15}$ Consider a substance possessing a nonlinear susceptibility with a spatial dependence. In this case, the output field, assuming "point sources" for the $u_{i}$ and using Eq. (10), is

$$
A_{3}\left(x_{0}, y_{0}, 0\right) \propto \mathscr{F}\left\{\chi_{\mathrm{NL}}{ }^{(3)}(x, y)\right\} .
$$

We thus have an output field that maps the spatial periodicities of the nonlinear optical components in a material. Hence the nonlinear structure of a medium (which, for example, might be homogeneous in its linear susceptibility) can be revealed.

In conclusion, the application of real-time image processing can be utilized both as a material probe and as a data-processing device. The use of four-wave nonlinear mixing in real-time convolution and correlation has been proposed and analyzed.

This research was supported by the Army Research Office, Durham, North Carolina. D. M. Pepper is a Hughes Research Laboratory Doctoral Fellow. D. Fekete is a Weizmann Institute, Israel, Postdoctoral Fellow.

D. M. Pepper acknowledges the support granted by the Hughes Aircraft Company. D. Fekete acknowledges the support granted by the Weizmann Institute of Israel.

\section{References}

1. A. B. Van der Lugt, IEEE Trans. Inform. Theory IT-10, 2 (1964).

2. J. W. Goodman, Introduction to Fourier Optics (McGraw-Hill, New York, 1968).

3. A. Yariv, Appl. Phys. Lett. 28, 89 (1976).

4. A. Yariv, J. Opt. Soc. Am. 66, 301 (1976).

5. R. A. Eremeeva, V. A. Kudryashov, I. N. Matrieev, T. G. Usacheva, and A. I. Chekemenev, Sov. J. Quantum Electron. 5, 1429 (1976).

6. R. W. Hellwarth, J. Opt. Soc. Am. 67, 1 (1977).

7. A. Yariv and D. M. Pepper, Opt. Lett. 1, 16 (1977).

8. P. D. Maker and R. W. Terhune, Phys. Rev. 137, A801 (1965).

9. See, for example, A. Yariv, Quantum Electronics (Wiley, New York, 1975), 2nd ed.

10. D. M. Pepper, D. Fekete, and A. Yariv, "Observation of amplified phase conjugate reflection and optical parametric oscillation by degenerate four-wave mixing in a transparent medium," submitted to Appl. Phys. Lett.; D. M. Bloom, P. F. Liao, and N. P. Economou, Opt. Lett. 2, 58 (1978).

11. D. M. Bloom and G. C. Bjorklund, Appl. Phys. Lett. 31, 592 (1977); S. M. Jensen and R. W. Hellwarth, Appl. Phys. Lett. 32, 166 (1978).

12. A. Yariv, Opt. Commun. 25, 23 (1978).

13. See, for example, B. Chen, E. Marom, and A. Lee, Appl. Phys. Lett. 31, 263 (1977), and references therein.

14. A. Yariv, J. AuYeung, D. Fekete, and D. M. Pepper, Appl. Phys. Lett. 32, 635 (1978).

15. R. W. Hellwarth and P. Christensen, Opt. Commun. 12, 318 (1974). 